\title{
BigData Express: Toward Predictable, Schedulable, and High-performance Data Transfer
}

Wenji Wu wenj@fnal.gov Internet2 Global Summit May 8, 2018 


\section{BigData Express}

- Funded by DOE's office of Advanced Scientific Computing Research (ASCR)

- Collaborative effort by Fermilab and Oak Ridge National Laboratory

- KISTI joined as a unfunded partner at 2017

- ESnet provides WAN service

- A three-year research project

苦Fermilab

- Start: Oct 1, 2015

- End: Sep 30, 2018

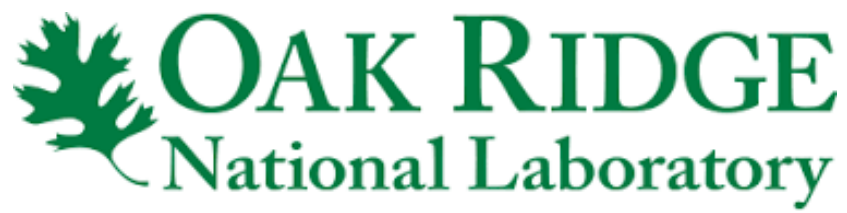

- http://bigdataexpress.fnal.gov 


\section{BigData Express Research Team}

- FNAL

- Wenji Wu (PI)

- Qiming Lu

- Liang Zhang

- Amy Jin

- Sajith Sasidharan

- Phil DeMar
- ORNL

- Nageswara Rao

- Gary Liu

- KISTI

- Syed Asif Shah

- Seo-Young Noh

- Jin Kim 


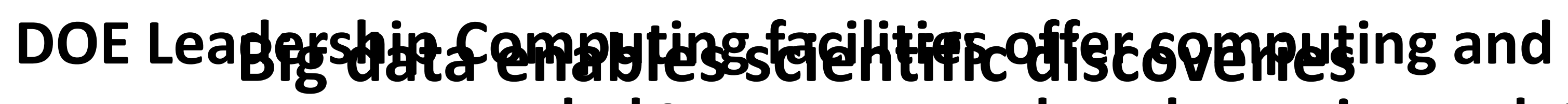
storage resources needed to process and analvze science data

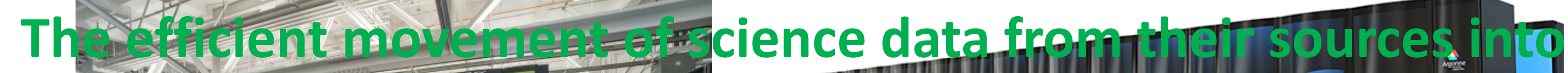

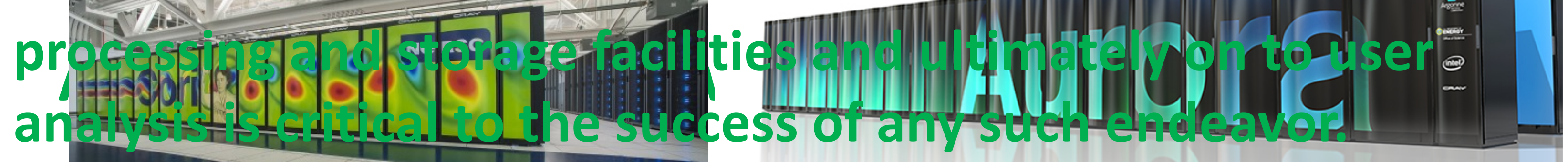

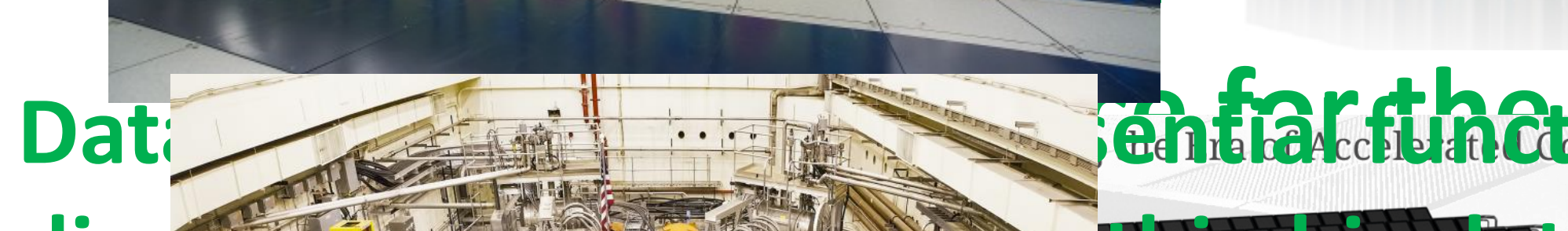
disc The Laroe Hadron Collider (LHC)

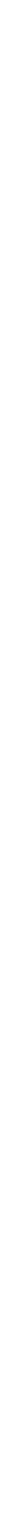




\section{Why BigData Express?}

- Targeted at optimizing data transfers in high-speed networks

- Large-scale data movement of Big Data Science

- High-speed network environments (40/100GE+)

- Builds on Multicore-Aware Data Transfer Middleware (MDTM)

- mdtmFTP: a high-performance data transfer tool

- Pipelined I/O-centric design to streamline data transfer

- MDTM optimizes use of underlying multicore system

- Extremely efficient in transferring of Lots Of Small Files (LOSF)

- http://mdtm.fnal.gov

- Orchestrates system (DTN), storage, \& network (SDN) resources

- To provide full end-to-end performance optimization 


\section{BigData Express versus SENSE}

- BigData Express is data transfer middleware

- Uses SENSE for WAN SDN services

- SENSE is a network service

- Provides higher-level applications with SDN-type services

- BigData Express is an application to SENSE

- BigData Express and SENSE are each stand-alone services in their own right

- BigData Express works fine without SENSE

- WAN component is simply Best Effort

- SENSE is agnostic to higher-level applications using its services 


\section{BigData Express Major Components}

- BDE Web Portal

- Allow users to access BigData Express data transfer services

- BDE Scheduler

- DTN as a service

- Co-scheduling of DTN, storage, and network

- BDE AmoebaNet

- Network as a service

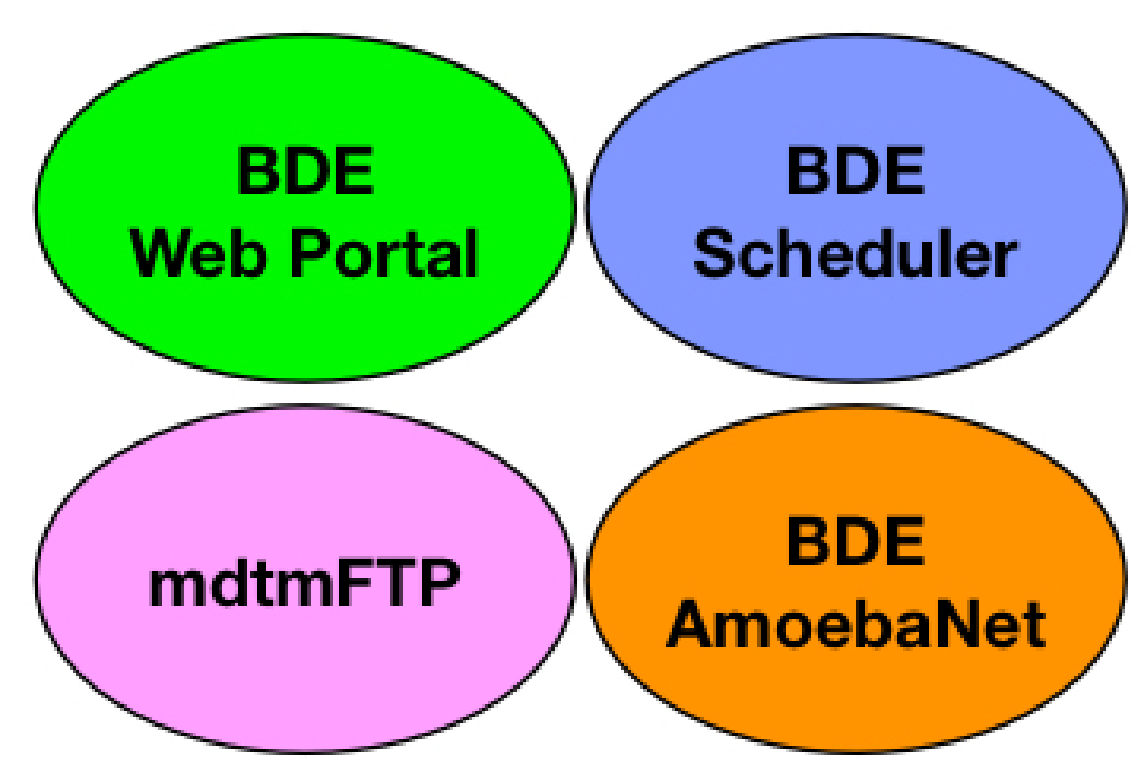

- mdtmFTP

- a high-performance data transfer engine

- http://mdtm.fnal.gov 


\section{BigData Express Major Components (cont.)}

- DTN Agents

- Manage and configure DTNs

- Collect and report the DTN configuration and status

- Storage Agents

- Manage and configure storage systems

- Data Transfer Launching Agent

- Launch data transfer jobs

- Support different data transfer protocols 


\section{BigData Express -- Distributed}

\section{Software Stack @ a BigData Express Site}

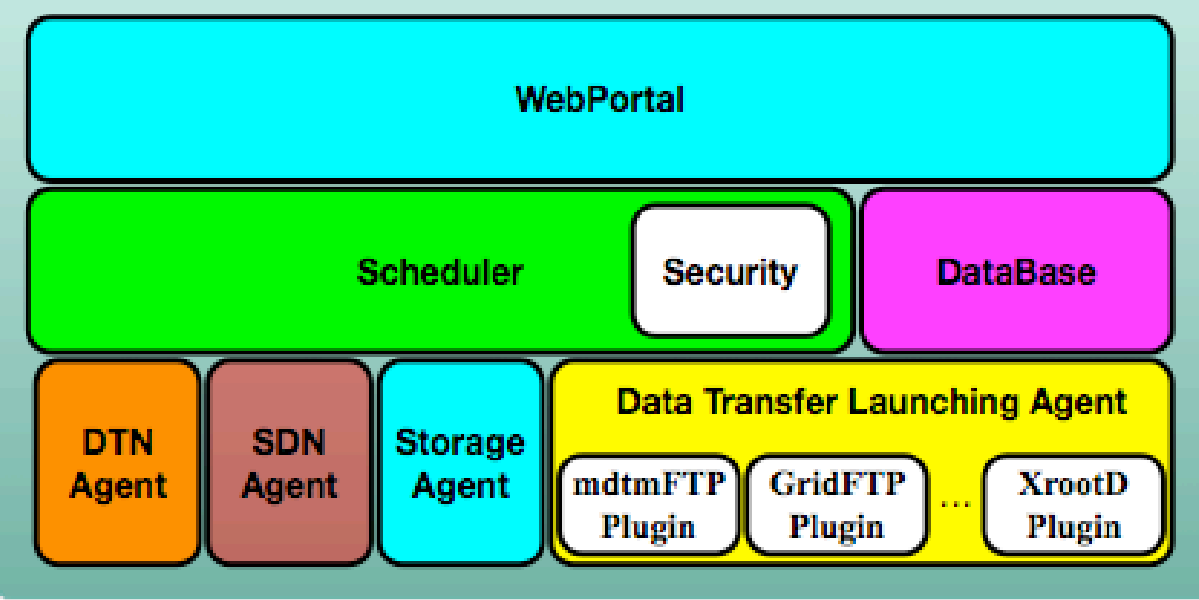

A Peer-to-Peer model
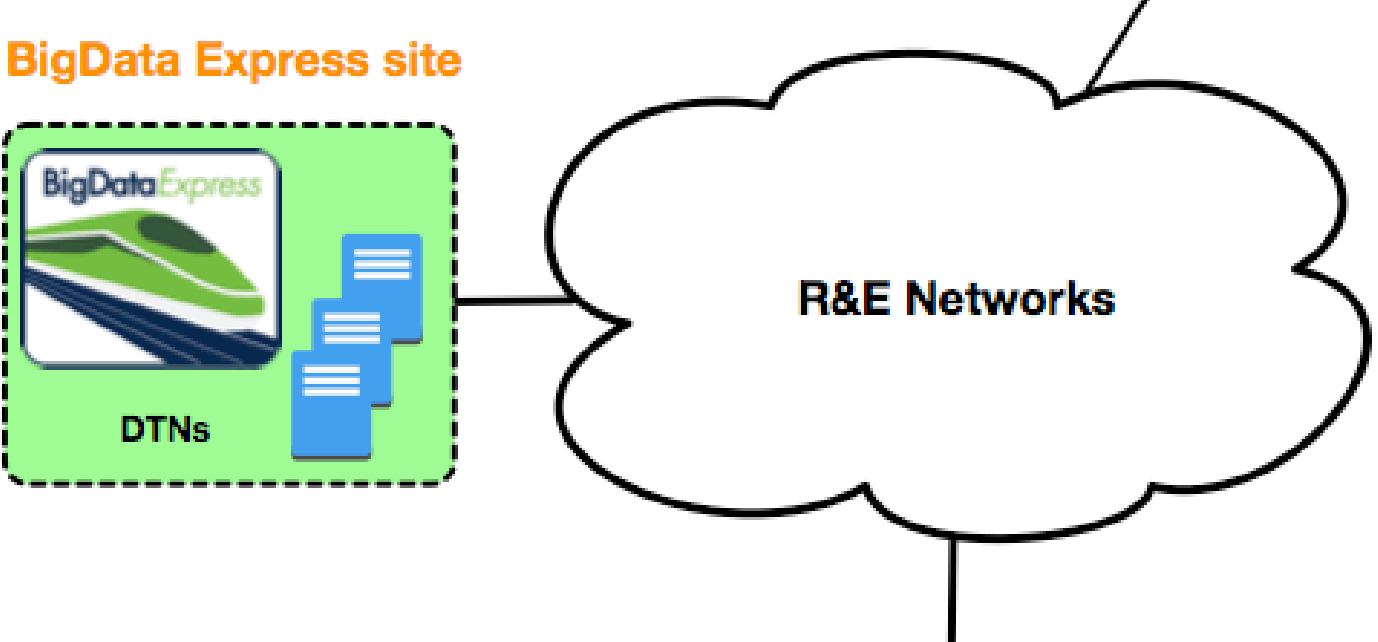

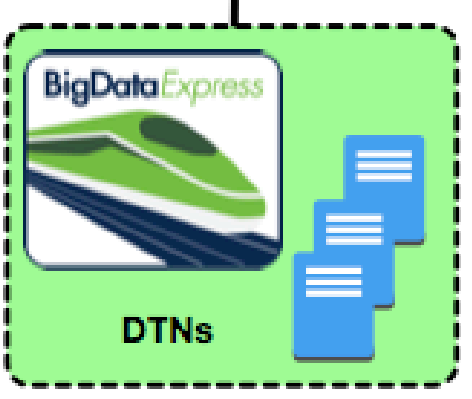

BigData Express site 


\section{BigData Express -- Flexible}

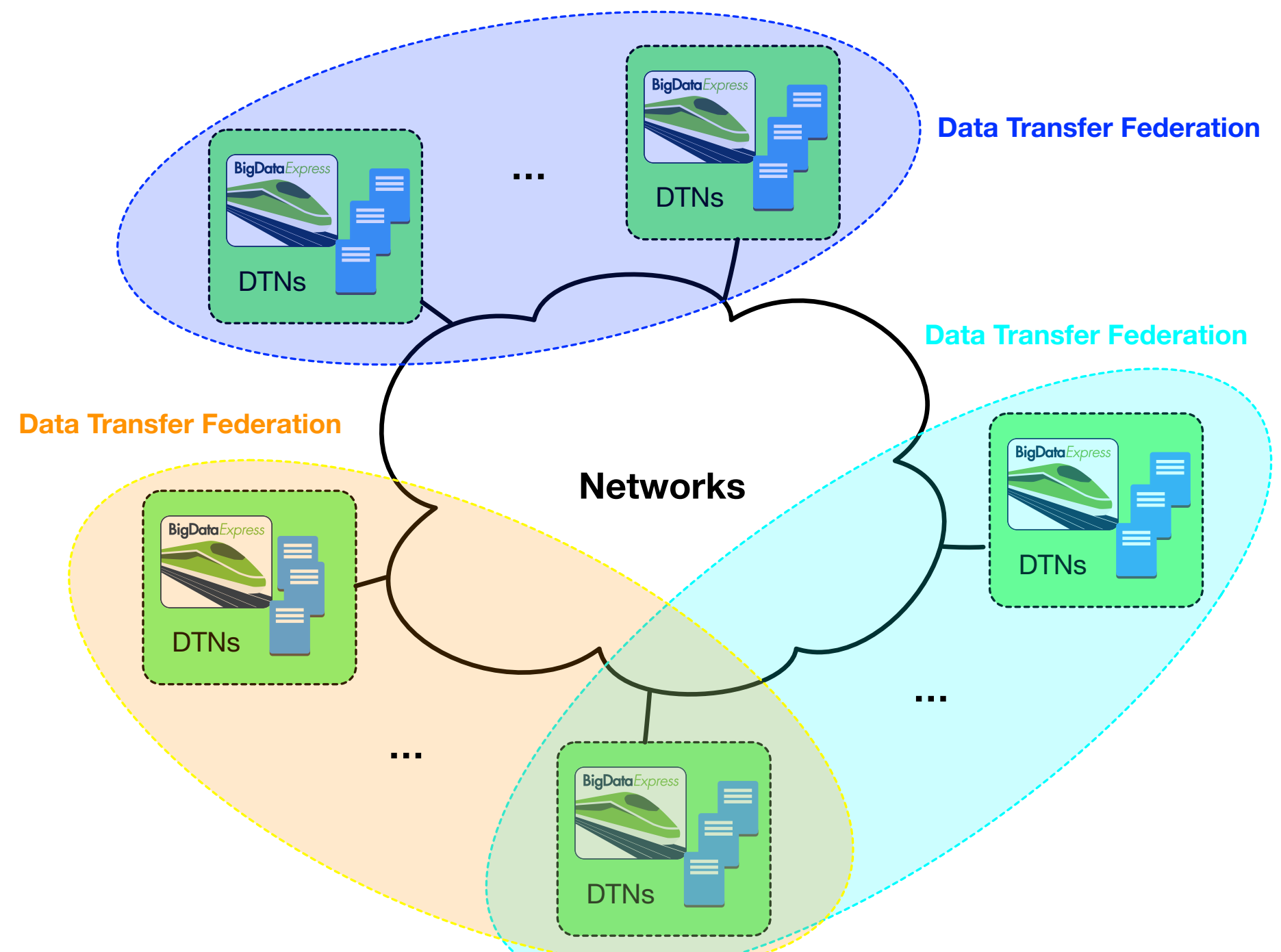

- Flexible to set up data transfer federations

- Providing inherent support for incremental deployment 


\section{BigData Express -- Scalable}

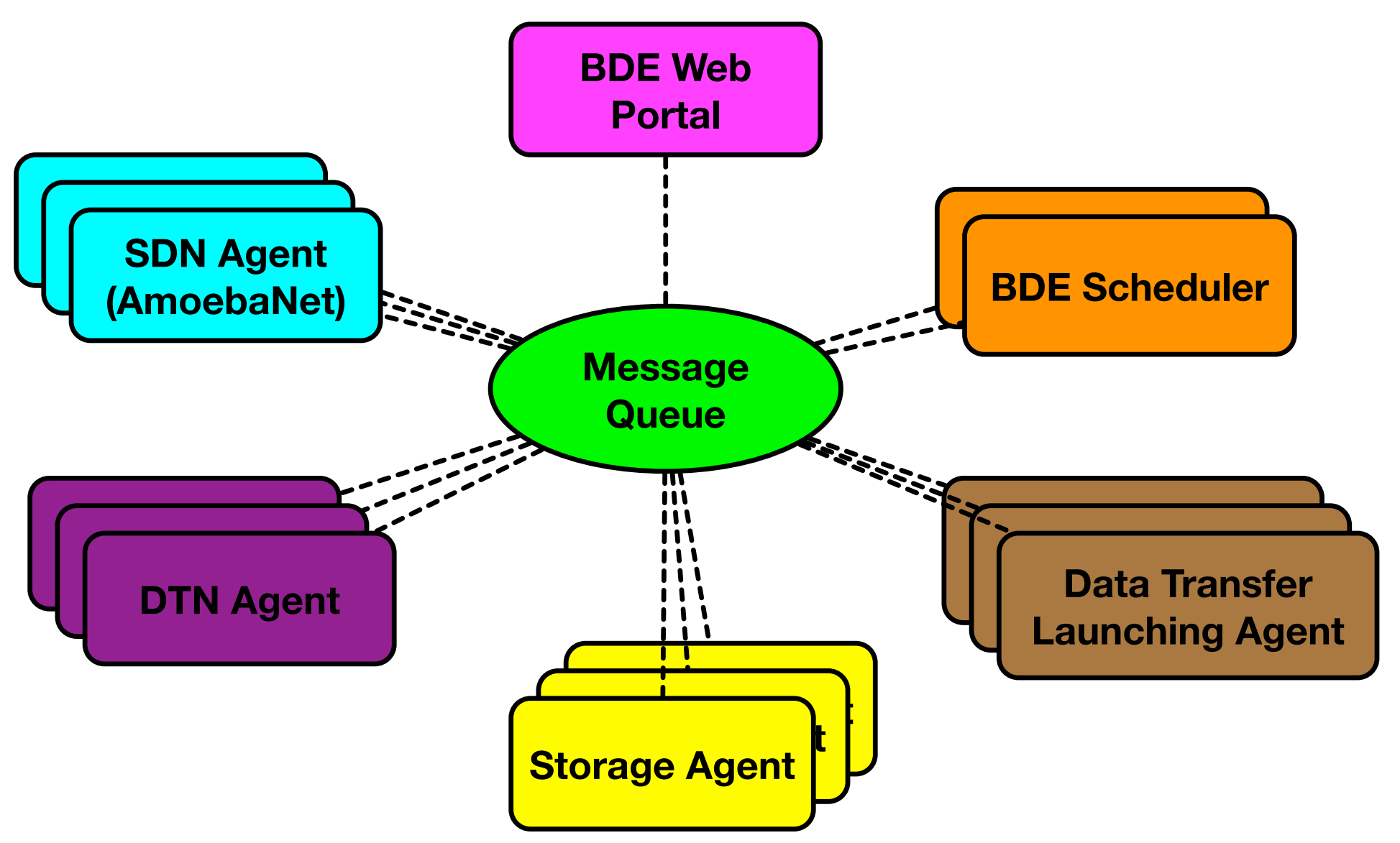

- BigData Express scheduler manages site resources through agents

- Use RabbitMQ as message bus 


\section{BigData Express -- Extensible}

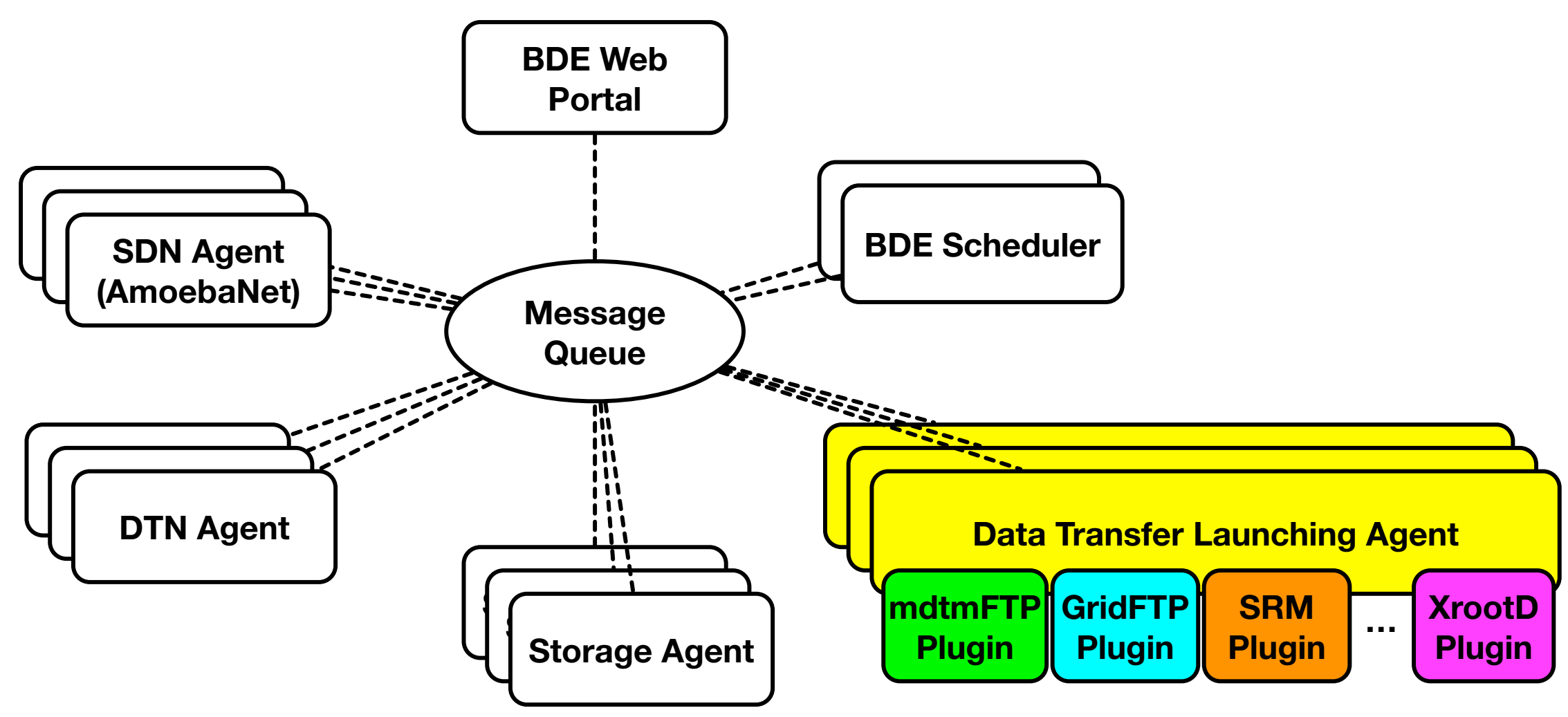

- Extensible Plugin framework to support various data transfer protocols - mdtmFTP, GridFTP, SRM, XrootD, ... 


\section{BigData Express -- End-to-End Data Transfer Model}

Site A - Smart E2E

Data Transfer Orchestrato

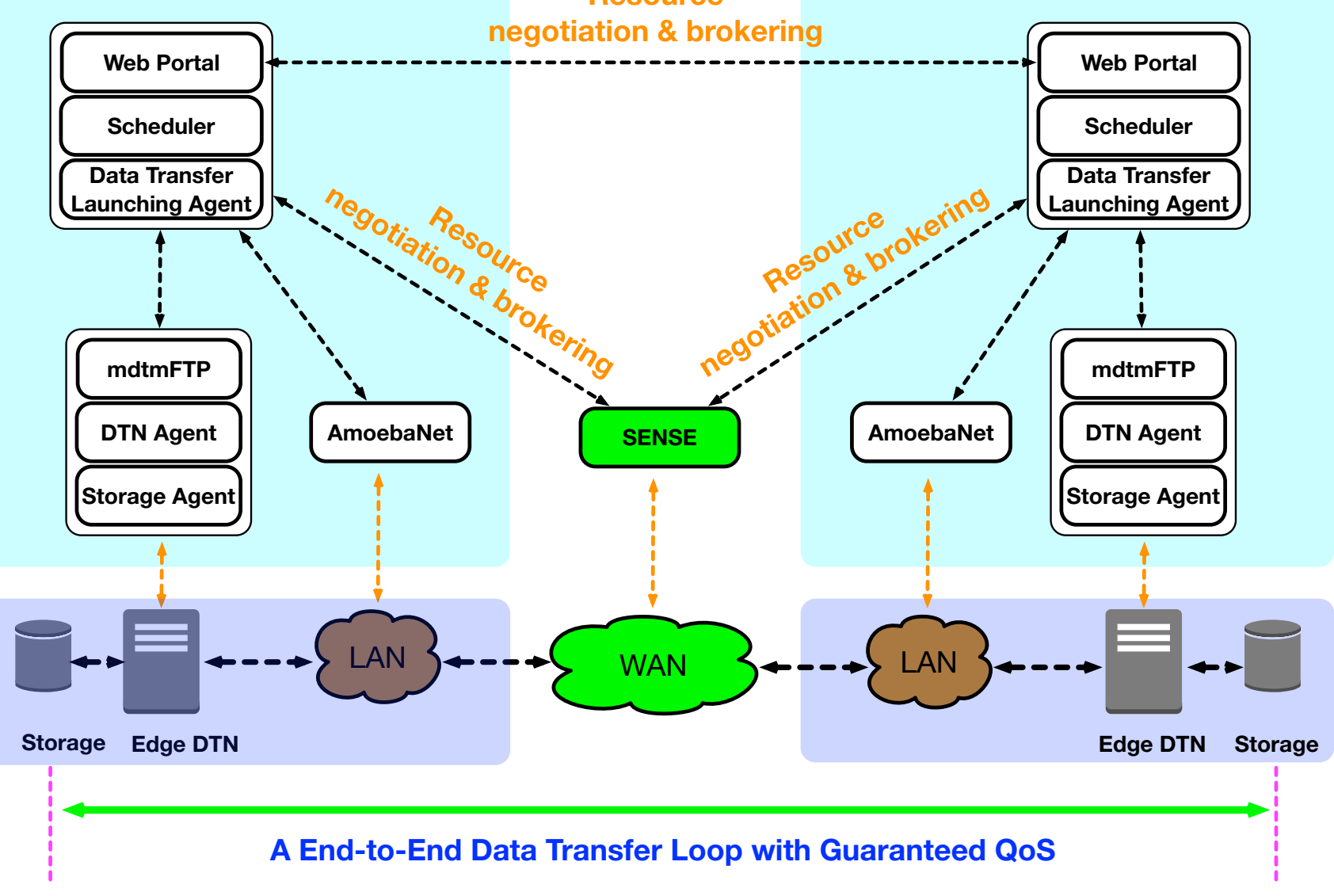

- Application-aware network service o On-demand programming

- Fast-provisioning of end-to-end network paths with guaranteed QoS

- Distributed resource negotiation \& brokering 


\section{BigData Express -- Three Types of Data Transfer}

- Real-time data transfer

- Deadline-bound data transfer

- Best-effort data transfer 


\section{BigData Express vs. Globus Online}

\begin{tabular}{|c|c|c|}
\hline Features & BigData Express & Globus Online \\
\hline Architecture & $\begin{array}{l}\text { - Distributed service } \\
\text { - Flexible to set up data transfer federations }\end{array}$ & - Centralized service \\
\hline Supported Protocols & $\begin{array}{l}\text { Extensible plugin framework to support multiple } \\
\text { protocols: } \\
\circ \text { mdtmFTP } \\
\circ \text { GridFTP, XrootD, SRM (coming soon) }\end{array}$ & - GridFTP \\
\hline SDN Support & $\begin{array}{l}\text { - Yes, Network as a service } \\
\text { - Fast-provisioning end-to-end network paths with } \\
\text { guaranteed QoS }\end{array}$ & - No \\
\hline Supported Data Transfers & $\begin{array}{l}\text { - Real-time data transfer } \\
\text { - Deadline-bound data transfer } \\
\text { - Best-effort data transfer }\end{array}$ & - Best-effort data transfer \\
\hline Error Handling & $\begin{array}{l}\text { - } \text { Checksum } \\
\text { - } \text { Retransmit }\end{array}$ & $\begin{array}{ll}\text { - } & \text { Checksum } \\
\text { - } & \text { Retransmit }\end{array}$ \\
\hline
\end{tabular}




\section{BigData Express SC'17 DEMO}

- BigData Express: a schedulable, predictable, and high-performance data transfer service

- QoS-guaranteed data transfer

- DTN as a service

- Network as a service

- Distributed resource brokering/matching 


\section{A Cross-Pacific SDN Testbed}

\section{茎Fermilab}

NaAK $\mathrm{RIDGE}$
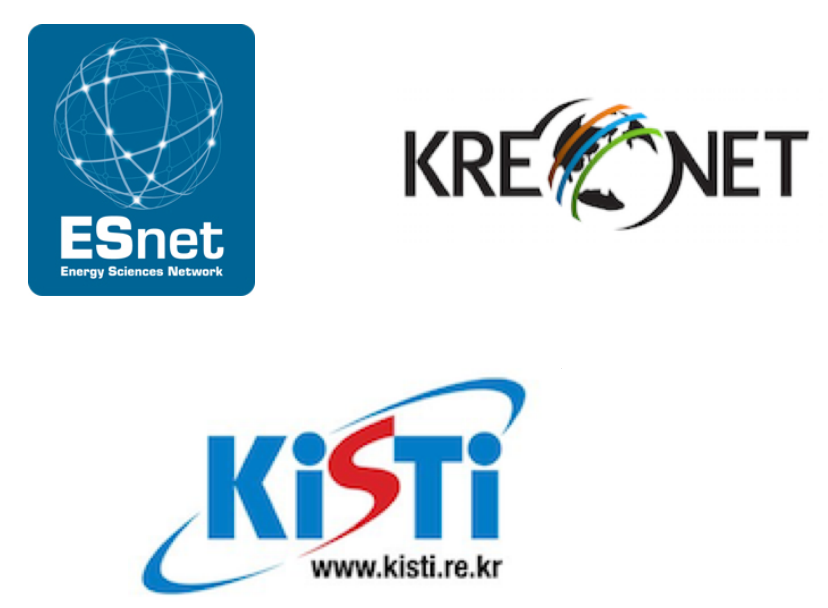

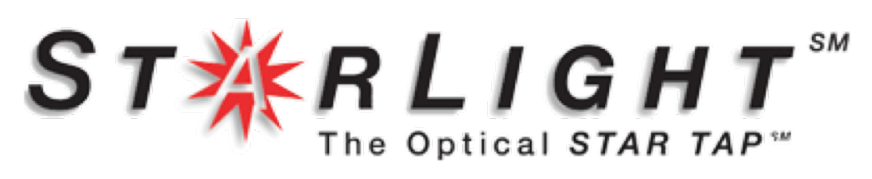

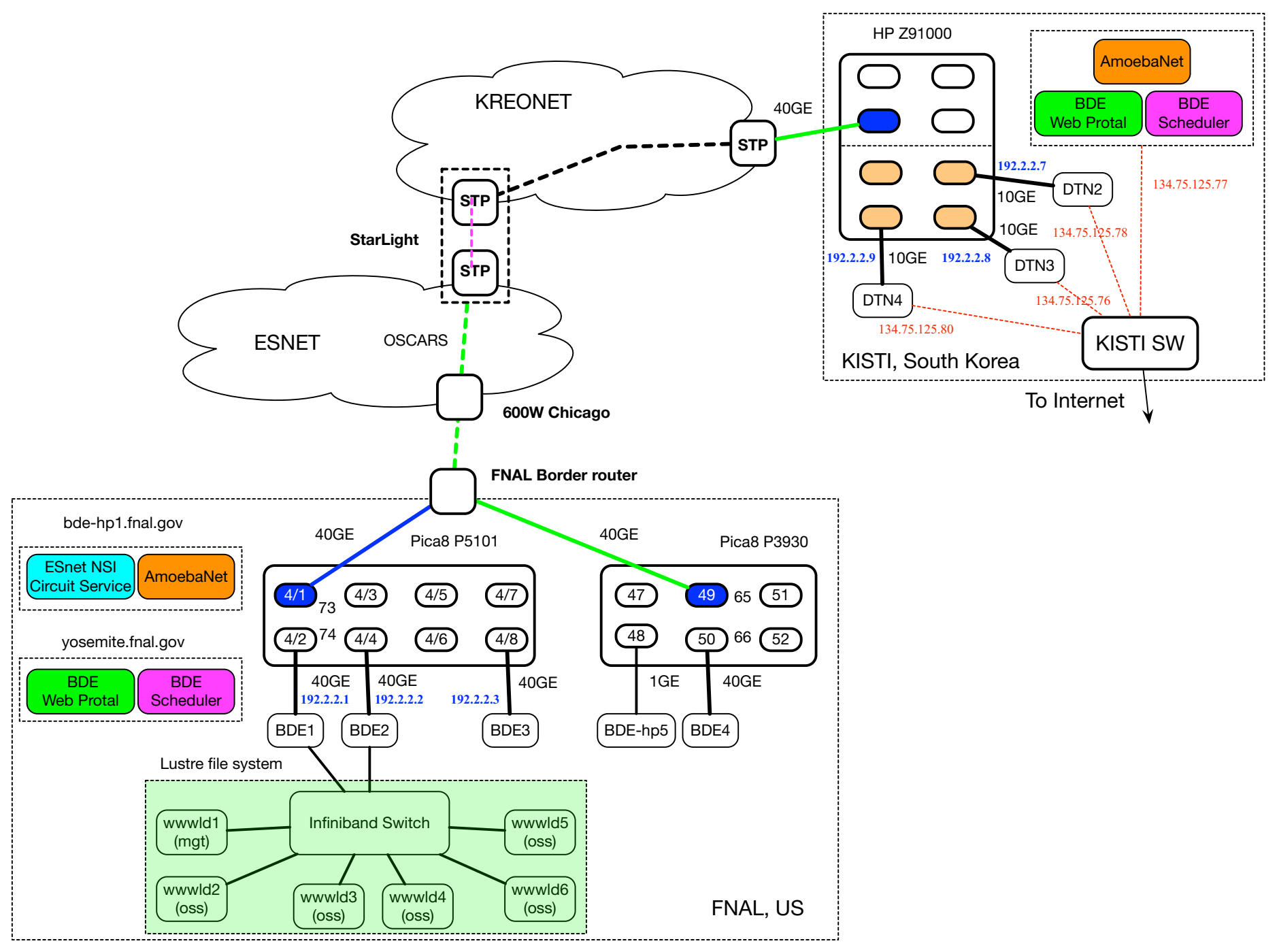




\section{BigData Express Deployment}

- Completed deployment: KISTI, UMD, StarLight, FNAL

- Ongoing deployment: KSTAR, ESnet

- Work with StarLight to deploy BDE at XRPs

- Pacific Research Platform (PRP)

- National Research Platform (NRP)

- Global Research Platform (GRP)

S T 海 $R$ L I G H T T

- The European Research Platform (ERP)

- Asia Research Platform (ARP)

- Collaborate with SENSE for BDE+SENSE deployment

- Work with US CMS to deploy BDE at US CMS sites

\section{萿 Fermilab}

ESnet

KiSTi

www.kisti.re.kr

MAX

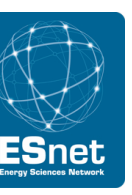




\section{Support Science}

- Fusion community

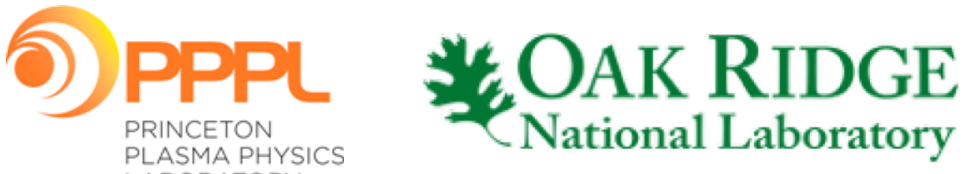

KSTAR

- Work with KSTAR, KISTI, PPPL, and ORNL to transfer/stream data from KSTAR to US research institutions

- XRPs (PRP, NRP, GRP, ERP, ARP)

S T棌 R L I G H T SDX $^{\prime \prime}$

- Work with StarLight to deploy BDE at XRPs to support various science

- HEP community

- Work with US CMS to deploy BDE at US CMS sites

- PI has been invited to give a BDE demo for US CMS

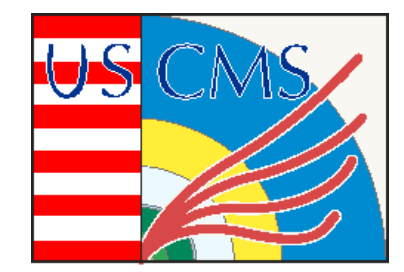

- Tentatively scheduled for the last week of May, 2018 
More information about BigData Express

\author{
http://bigdataexpress.fnal.gov
}

PI: Wenji Wu, Fermilab

wenji@fnal.gov 